\title{
Effect of toe-spread-out exercise on hallux valgus angle and cross-sectional area of abductor hallucis muscle in subjects with hallux valgus
}

\author{
Moon-Hwan Kim, PT, MSc ${ }^{1)}$, Chung-Hwi Yi, PT, PhD ${ }^{2)}$, Jong-Hyuck Weon, PT, PhD ${ }^{3)}$, \\ Heon-Seock Cynn, PT, PhD ${ }^{2)}$, Do-Young Jung, PT, PhD ${ }^{3)}$, Оh-Yun Kwon, PT, PhD $\left.^{4}\right)^{*}$ \\ 1) Department of Physical Therapy, Graduate School, Yonsei University, Republic of Korea \\ 2) Department of Physical Therapy, College of Health Science, Yonsei University, Republic of Korea \\ 3) Department of Physical Therapy, College of Tourism and Health Science, Joongbu University, \\ Republic of Korea \\ 4) Department of Physical Therapy, College of Health Science, Laboratory of Kinetic Ergocise Based on \\ Movement Analysis, Yonsei University: 1 Yonseidae-gil, Wonju, Gangwon-do 220-710, Republic of Korea
}

\begin{abstract}
Purpose] This study investigated whether the toe-spread-out exercise affects the hallux valgus angle, the cross-sectional area of the abductor hallucis muscle, and the hallux valgus angle during active abduction. [Subjects and Methods] Twenty-four subjects with hallux valgus were randomly assigned to orthosis and orthosis plus toe-spread-out exercise groups. The orthosis group wore the orthosis for 8 weeks, while the orthosis plus toespread-out group also performed the toe-spread-out exercise. The hallux valgus angle, the cross-sectional area of the abductor hallucis muscle, and the hallux valgus angle during active abduction were measured initially and after 8 weeks by radiography and ultrasonography. [Results] While there were no significant changes in the three parameters in the orthosis group, there were significant differences in the orthosis plus toe-spread-out exercise group after 8 weeks. In addition there were significant differences in the three measures between the two groups. [Conclusion] The toe-spread-out exercise reduces the hallux valgus angle and hallux valgus angle during active abduction, and increases the cross-sectional area of the abductor hallucis muscle. The toe-spread-out exercise is recommended for patients with mild to moderate hallux valgus.

Key words: Hallux valgus, Radiography, Toe-spread-out exercise
\end{abstract}

(This article was submitted Oct. 5, 2014, and was accepted Nov. 28, 2014)

\section{INTRODUCTION}

Hallux valgus (HV) is defined as a progressive abnormality in the degree of lateral deviation of the big toe at the metatarsophalangeal (MTP) joint, which may involve pain at the medial aspect of the first metatarsal head ${ }^{1)}$. The HV angle, which corresponds to the angle between the bisection line of the first metatarsal bone and the first proximal phalan $x^{2)}$, is classified as either normal ( $\leq 15$ degrees), mild ( $<20$ degrees), moderate (20-40 degrees), or severe $(\geq 40$ degrees) $)^{3)}$.

Generally, available treatments for HV are divided into operative and non-operative treatments. Although the operative method is effective for mild-to-moderate HV, pain persists for weeks or even months afterward, and necessitates protracted non-weight-bearing periods ${ }^{4}, 5$ ). Non-operative

\section{*Corresponding author. Oh-Yun Kwon (E-mail: kwonoy@} yonsei.ac.kr)

(C2015 The Society of Physical Therapy Science. Published by IPEC Inc. This is an open-access article distributed under the terms of the Creative Commons Attribution Non-Commercial No Derivatives (by-ncnd) License $<$ http://creativecommons.org/licenses/by-nc-nd/3.0/>. methods include wearing an orthosis and engaging in specific exercises. The effectiveness of orthosis is subject to conjecture. Several studies report there is no benefit of orthosis on big toe angle ${ }^{6,7)}$, but other studies have suggested that orthoses are effective for HV surgery patients, and can confer short-term symptomatic relief ${ }^{4,8}$.

Specific exercises can also be used to treat HV. Several researchers have suggested that exercise is necessary during the early stages of $\mathrm{HV}$, to prevent further increases in the $\mathrm{HV}$ angle ${ }^{9,10)}$. HV patients are characterized by an imbalance in the activities of the abductor hallucis $(\mathrm{AbdH})$ and adductor hallucis (AddH) muscles $\left.{ }^{9}, 11\right)$. Several studies have highlighted the importance of strengthening the $\mathrm{AbdH}$ muscle in $\mathrm{HV}$ patients ${ }^{9,11)}$, but few studies have determined which specific exercises should be performed. Recently, Keller ${ }^{12)}$ introduced a novel exercise, the "Toe-Spread-Out" (TSO) exercise, and a subsequent electromyographic (EMG) study revealed greater activation of the $\mathrm{AbdH}$ muscle during performance of the TSO exercise than during the short-foot exercise, in mild HV patients ${ }^{13}$. However, evidence of the long-term effectiveness of the TSO exercise is currently lacking, and although there have been many EMG studies of the AbdH muscle activity ${ }^{11,13-16)}$, few have measured the cross-sectional area (CSA) of the AbdH muscle at pre- and 
post-intervention. Therefore, we aimed to determine whether the TSO exercise is effective at decreasing the HV angle, the $\mathrm{HV}$ angle during active abduction, and increasing the CSA of the AbdH muscle, using an 8-week randomized controlled trial design. We hypothesized that wearing an orthosis, in conjunction with performing TSO exercises, would decrease the $\mathrm{HV}$ angle, and the $\mathrm{HV}$ angle during active abduction, and increase the CSA of the AbdH muscle.

\section{SUBJECTS AND METHODS}

Twenty-four subjects (13 males, 11 females) between 19 and 29 years of age, with a diagnosis of HV, were included. Fifteen subjects were categorized as having a mild $\mathrm{HV}$ angle, and nine as having a moderate HV angle, during standing. The only inclusion criterion was an HV angle of $>15$ degrees. Exclusion criteria included a diagnosis of rheumatoid arthritis or osteoarthritis, previous history of foot surgery, previous use of a foot orthosis, and previously diagnosed central or peripheral nervous system injury. Subjects were randomly divided into two groups of 12 persons each using the block randomization method. Subjects in the experimental group were trained in the TSO exercise and required to wear an orthosis. Control subjects were only required to wear an orthosis. All subjects signed an informed consent form approved by the YONSEI University Wonju Institutional Review Board for Human Investigations prior to participation.

The HV angles of subjects were measured by dorsoplantar radiography (KOB, Donga X-ray, Anyang, Korea) in a standing position, with normal alignment. An experienced radiologist measured the $\mathrm{HV}$ angle using the Centricity PACS RA1000 software package (ver. 2.1.0; GE Healthcare Integrated IT Solutions, Barrington, USA). The HV angle was measured as the angle between the bisection line of the first metatarsal bone and the first proximal phalan $\mathrm{x}^{2}$.

For the measurement of the HV angle during active abduction of the hallux, subjects sat on a chair situated above the radiographic table, and assumed a 90 degrees flexion angle between the hip and knee, with the ankle maintained in the neutral position (i.e., 0 degree). Dorsoplantar radiographs were taken at rest, and during maximal abduction of the big toe. These radiographs were analyzed in the same way as for the HV angle.

The CSA of the AbdH muscle was measured using ultrasound (SonaAce X8, Medison Co Ltd., Seoul, Korea), with subjects relaxed in the long-seated position. The measured foot was positioned with the ankle in the neutral position and the knee flexed at 15 degrees. The hip joint was maintained at a comfortable degree of external rotation, for ease of measurement during the $\mathrm{AbdH}$ muscle scan ${ }^{17}$. The researcher palpated the medial malleolus and used a ruler to draw a line anterior to this bony landmark, in an inferior direction. The probe was positioned perpendicular to the drawn line. Three images of the left foot were obtained, and the mean CSA of the AbdH muscle was calculated. The ultrasound procedure was conducted by an experienced doctoral graduate student. The CSA of the AbdH muscle was obtained to enable manual measurement of the muscle ${ }^{17)}$. The CSA of the $\mathrm{AbdH}$ muscle was measured three times, and the mean
Table 1. Descriptive data of the subjects of this study $(\mathrm{N}=24)$

\begin{tabular}{lcc}
\hline Parameter & $\begin{array}{c}\text { Orthosis plus } \\
\text { TSO exercise } \\
\text { group }(\mathrm{n}=12)\end{array}$ & $\begin{array}{c}\text { Orthosis group } \\
(\mathrm{n}=12)\end{array}$ \\
\hline Age (years) & $22.2 \pm 2.04$ & $22.8 \pm 2.83$ \\
Height $(\mathrm{cm})$ & $169.4 \pm 9.01$ & $168.3 \pm 8.27$ \\
Weight $(\mathrm{kg})$ & $62.6 \pm 12.03$ & $64.5 \pm 13.89$ \\
HV angle $\left(^{\circ}\right)$ & $18.3 \pm 3.42$ & $19.3 \pm 3.25$ \\
CSA of AbdH muscle $\left(\mathrm{cm}^{2}\right)$ & $2.0 \pm 0.43$ & $2.0 \pm 0.61$ \\
HV angle during active & $15.2 \pm 6.64$ & $17.3 \pm 5.97$ \\
abduction $\left({ }^{\circ}\right)$ & &
\end{tabular}

TSO: toe-spread-out; HV: hallux valgus; CSA: cross-sectional area; $\mathrm{AbdH}$ : abductor hallucis. Values are mean \pm standard deviation. *Independent t-test, $\mathrm{p}<0.05$.

value was calculated.

The 24 subjects were randomly divided into two groups (orthosis and orthosis plus TSO exercise). The orthosis group was required only to wear the orthosis; and the orthosis plus TSO exercise group wore the orthosis and also performed the TSO exercise for a period of 8 weeks. Each subject wore an appropriately sized orthosis, made of spandex material, the Bunion Sleeve (DLI Co. Seoul, Korea). Subjects were required to wear the orthosis for $>8 \mathrm{~h} /$ day. Subjects in the orthosis plus TSO exercise group were trained to perform the TSO exercise following the method described by Keller ${ }^{12)}$. The subjects were instructed to lift all toes while keeping the metatarsal heads and heel on the floor, and to then push the little toe downward, in a lateral direction, while pushing the big toe slowly downward in a medial direction. Prior to the commencement of the experiment, training exercises were performed for 2 days, for a total of 2 hours. Between the first experimental day and the end of the study, subjects performed the TSO exercise for $20 \mathrm{~min} /$ day, 4 days/week. On a single occasion each week, the orthosis plus TSO exercise group received 30 minutes of instruction from a supervisor regarding correct performance of the TSO exercise. All subjects were evaluated before and after the study, with respect to $\mathrm{HV}$ angle, CSA of the $\mathrm{AbdH}$ muscle, and $\mathrm{HV}$ angle during active abduction.

Data are expressed as means \pm standard deviation. The independent t-test was used to assess the significance of between-group differences in the initial value of the HV angle, the CSA of the $\mathrm{AbdH}$ muscle, and the HV angle during active abduction. The independent t-test was also used to investigate group differences after the 8-week intervention. The paired t-test was used to determine the pre- and postintervention group differences. Statistical analyses were performed using the SPSS version 21.0 for Windows software package (SPSS, Inc., Chicago, IL, USA). A value of $\mathrm{p}<0.05$ was considered to indicate statistical significance.

\section{RESULTS}

The general characteristics of subjects, including the HV angle, CSA of the $\mathrm{AbdH}$ muscle, and the $\mathrm{HV}$ angle during active abduction, did not differ between the groups at baseline (Table 1). 
Table 2. Comparison of orthosis plus TSO exercise group and orthosis group between pre- and post-intervention ( $\mathrm{N}=12)$

\begin{tabular}{lcccc}
\hline \multirow{2}{*}{ Parameter } & \multicolumn{2}{c}{ Orthosis plus TSO exercise group } & \multicolumn{2}{c}{ Orthosis group } \\
\cline { 2 - 5 } & Baseline & 8 weeks & Baseline & 8 weeks \\
\hline $\mathrm{HV}$ angle $\left(^{\circ}\right)$ & $18.33 \pm 3.42$ & $14.92 \pm 3.36^{*}$ & $19.25 \pm 3.25$ & $18.75 \pm 3.52$ \\
$\mathrm{CSA}$ of $\mathrm{AbdH}\left(\mathrm{cm}^{2}\right)$ & $2.04 \pm 0.43$ & $2.52 \pm 0.66^{*}$ & $2.04 \pm 0.62$ & $1.93 \pm 0.51$ \\
$\mathrm{HV}$ angle during active abduction $\left(^{\circ}\right)$ & $15.17 \pm 6.64$ & $8.75 \pm 4.31^{*}$ & $17.00 \pm 5.95$ & $16.17 \pm 5.22$ \\
\hline
\end{tabular}

TSO: toe-spread-out; HV: hallux valgus; CSA: cross-sectional area; AbdH: abductor hallucis, Values are mean \pm standard deviation. "Paired t-test, $\mathrm{p}<0.05$.

Table 3. Comparison of difference between orthosis group and orthosis plus TSO exercise group $(\mathrm{N}=24)$

\begin{tabular}{lcc}
\hline Parameter & $\begin{array}{c}\text { Orthosis plus TSO } \\
\text { exercise group }(\mathrm{n}=12)\end{array}$ & Orthosis group $(\mathrm{n}=12)$ \\
\hline $\mathrm{HV}$ angle $\left(^{\circ}\right)$ & $-3.41 \pm 3.17^{*}$ & $-0.5 \pm 2.07$ \\
$\mathrm{CSA}$ of $\mathrm{AbdH}\left(\mathrm{cm}^{2}\right)$ & $0.48 \pm 0.28^{*}$ & $-0.11 \pm 0.34$ \\
HV angle during active abduction $\left(^{\circ}\right)$ & $-6.42 \pm 3.42^{*}$ & $-0.83 \pm 2.92$ \\
\hline
\end{tabular}

TSO: toe-spread-out; HV: hallux valgus; CSA: cross-sectional area; AbdH: abductor hallucis, Values are mean \pm standard deviation. ${ }^{*}$ Independent t-test $\mathrm{p}<0.05$

After 8 weeks, there was a significant decrease in the $\mathrm{HV}$ angle, and the HV angle during active abduction, and a significant increase in the CSA of the AbdH muscle, in the orthosis plus TSO exercise group compared with the baseline values. No significant differences from baseline in the HV angle, CSA of the AbdH muscle, and the HV angle during active abduction were observed in the orthosis group (Table 2).

There were significant group differences in HV angle, CSA of the AbdH muscle, and HV angle during active abduction after the 8-week intervention $(\mathrm{p}<0.05$; Table 3$)$.

The pre- and post-intervention effect sizes, in the orthosis plus TSO exercise and orthosis groups, were respectively 1.01 and 0.15 for the HV angle, 0.86 and 0.20 for the CSA of the $\mathrm{AbdH}$, and 1.15 and 0.15 for the HV angle during active abduction. The difference of between-group after 8-week intervention in effect sizes, for $\mathrm{HV}$ angle, CSA of the $\mathrm{AbdH}$, and $\mathrm{HV}$ angle during active abduction, were $1.09,1.89$, and 1.76 , respectively.

\section{DISCUSSION}

After the 8-week intervention, the HV angle, and the HV angle during active abduction, were significantly reduced, and the CSA of the AbdH muscle was significantly increased in the orthosis plus TSO exercise group, but there were no significant differences in the orthosis group.

The AbdH muscle originates in the medial process of the calcaneal tuberosity, flexor retinaculum, plantar aponeurosis, and adjacent intermuscular septum, and connects to the medial side of the base of the proximal phalanx of the big toe $^{18)}$. The AbdH muscle also sends lateral fibers to the medial tendon of the flexor brevis ${ }^{19)}$, and is involved not only in abduction but also in plantar flexion of the first MTP joint ${ }^{20)}$. Therefore, exercises targeting the AbdH muscle should involve diagonal movements combining both abduction and flexion of the big toe. In a previous study, TSO exercises promoted greater $\mathrm{AbdH}$ muscle activity; i.e., abduction and flexion of the big toe, compared with the short-foot exercise, thereby strengthening the $\mathrm{AbdH}$ muscle in mild $\mathrm{HV}$ subjects $^{13)}$. Therefore, we employed the TSO exercise in this study. After performing the TSO exercise for 8 weeks, the othosis plus TSO exercise group showed a significant decrease of 3.41 degrees in the average HV angle.

In the orthosis plus TSO exercise group, the CSA of the AbdH muscle was significantly increased after 8 weeks of treatment, but no change was observed in the orthosis group. Therefore, it appears that the TSO exercise was responsible for the increased CSA of the $\mathrm{AbdH}$ muscle, which in turn affects muscular function and force ${ }^{21)}$. Stewart et al. ${ }^{17)}$ assessed the relationship between the CSA of the AbdH muscle and the severity of HV using musculoskeletal ultrasound, and reported a significantly larger CSA in non-HV than in $\mathrm{HV}$ patients. The CSA of the AbdH muscle is important, because muscle size is considered an important determinant of muscle strength, and Maughan ${ }^{22}$, demonstrated a positive correlation between muscle strength and CSA. In our study, TSO exercise increased the CSA of AbdH muscle, and decreased the $\mathrm{HV}$ angle, and the HV angle during active abduction in the orthosis plus TSO exercise group. Because the TSO exercise involves abduction of the big toe, it may have a positive impact on the CSA of $\mathrm{AbdH}$ and $\mathrm{HV}$ angle during active abduction.

As yet, the effects of orthosis on HV patients are subject to conjecture. This study showed that there were no statistically significant differences in the three parameters after the 8 -week intervention in the orthosis group. These results are similar to the results of previous studies, which found there was no decrease in the HV angle ${ }^{7,23)}$.

The present study had several limitations. First, the results cannot be generalized because our subjects were relatively young (mean age $=23$ years), and had only mild- 
to-moderate HV. Additional research examining the effects of TSO exercise on different age groups, and in severe HV cases, is required. Second, we didn't evaluate the strength of the AbdH muscle during the 8 weeks. Future studies should assess the strength of AbdH muscle after TSO exercise lasting for 8 weeks.

\section{REFERENCES}

1) Sarwark JF: Essentials of Musculoskeletal Care, 4th ed. Illinois: American Academy of Orthopaedic Surgeons, 2010.

2) Coughlin MJ, Saltzman CL, Nunley JA 2nd: Angular measurements in the evaluation of hallux valgus deformities: a report of the ad hoc committee of the American Orthopaedic Foot \& Ankle Society on angular measurements. Foot Ankle Int, 2002, 23: 68-74. [Medline]

3) Mann RA, Coughlin MJ: Adult hallux valgus. In: Mann RA, Coughlin M (eds): Surgery of the foot and ankle, 7th ed. St. Louis: Mosby Yearbook, 1999.

4) Torkki M, Malmivaara A, Seitsalo S, et al.: Surgery vs orthosis vs watchful waiting for hallux valgus: a randomized controlled trial. JAMA, 2001, 285: 2474-2480. [Medline] [CrossRef]

5) Wülker N, Mittag F: The treatment of hallux valgus. Dtsch Arztebl Int, 2012, 109: 857-867, quiz 868. [Medline]

6) Ferrari J, Higgins JP, Prior TD: Interventions for treating hallux valgus (abductovalgus) and bunions. Cochrane Database Syst Rev, 2004, 1 CD000964. [Medline]

7) Tehraninasr A, Saeedi H, Forogh B, et al.: Effects of insole with toe-separator and night splint on patients with painful hallux valgus: a comparative study. Prosthet Orthot Int, 2008, 32: 79-83. [Medline] [CrossRef]

8) Unver B, Sampiyon O, Karatosun V, et al.: Postoperative immobilisation orthosis for surgically corrected hallux valgus. Prosthet Orthot Int, 2004, 28: 278-280. [Medline]

9) Arinci Incel N, Genç H, Erdem HR, et al.: Muscle imbalance in hallux valgus: an electromyographic study. Am J Phys Med Rehabil, 2003, 82:
345-349. [Medline] [CrossRef]

10) Groiso JA: Juvenile hallux valgus. A conservative approach to treatment. J Bone Joint Surg Am, 1992, 74: 1367-1374. [Medline]

11) Shimazaki $K$, Takebe $K$ : Investigations on the origin of hallux valgus by electromyographic analysis. Kobe J Med Sci, 1981, 27: 139-158. [Medline]

12) Keller D: Yoga Plus Joyful Living Magazine. November-December 2008, $72-79$.

13) Kim MH, Kwon OY, Kim SH, et al.: Comparison of muscle activities of abductor hallucis and adductor hallucis between the short foot and toespread-out exercises in subjects with mild hallux valgus. J Back Musculoskeletal Rehabil, 2013, 26: 163-168. [Medline]

14) Heo HJ, An DH: The effect of an inclined ankle on the activation of the abductor hallucis muscle during short foot exercise. J Phys Ther Sci, 2014, 26: 619-620. [Medline] [CrossRef]

15) Heo HJ, Koo YM, Yoo WG: Comparison of selective activation of the abductor hallucis during various exercises. J Phys Ther Sci, 2011, 23: $915-$ 918. [CrossRef]

16) Lee JE, Park GH, Lee YS, et al.: A comparison of muscle activities in the lower extremity between flat and normal feet during one-leg standing. J Phys Ther Sci, 2013, 25: 1059-1061. [Medline] [CrossRef]

17) Stewart S, Ellis R, Heath M, et al.: Ultrasonic evaluation of the abductor hallucis muscle in hallux valgus: a cross-sectional observational study. BMC Musculoskelet Disord, 2013, 14: 45. [Medline] [CrossRef]

18) Kendall FP, McCreary EK, Provance PG, et al.: Muscles: Testing and Function with Posture and Pain, 5th ed. Baltimore: Williams \& Wilkins, 2005.

19) Thomson SA: Hallux varus and metatarsus varus: a five-year study (19541958). Clin Orthop, 1960, 16: 109-118. [Medline]

20) Brenner E: Insertion of the abductor hallucis muscle in feet with and without hallux valgus. Anat Rec, 1999, 254: 429-434. [Medline] [CrossRef]

21) Lieber RL: Skeletal muscle structure and function. Baltimore: Williams \& Wilkins, 1992.

22) Maughan RJ, Watson JS, Weir J: Strength and cross-sectional area of human skeletal muscle. J Physiol, 1983, 338: 37-49. [Medline] [CrossRef]

23) Macfarlane A, Kilmartin T: Conservative treatment of juvenile hallux valgus - a seven year prospective study. Br J Pod, 2004, 4: 101-105. 